\title{
A Simple Model to Assess the Probability of Invasion in Ductal Carcinoma In Situ of the Breast Diagnosed by Needle Biopsy
}

\author{
Oldřich Coufal, ${ }^{1,2}$ Iveta Selingerová, ${ }^{3}$ Pavlína Vrtělová, ${ }^{1}$ Petr Krsička, ${ }^{1}$ \\ Lucie Gabrielová, ${ }^{1}$ Pavel Fabian, ${ }^{4}$ Kateřina Stískalová, ${ }^{5}$ Monika Schneiderová, ${ }^{5}$ \\ Alexandr Poprach, ${ }^{2}$ and Ivan Justan ${ }^{1}$ \\ ${ }^{1}$ Department of Surgical Oncology, Masaryk Memorial Cancer Institute, Zluty kopec 7, 65653 Brno, Czech Republic \\ ${ }^{2}$ Department of Comprehensive Cancer Care, Masaryk Memorial Cancer Institute, Zluty kopec 7, 65653 Brno, Czech Republic \\ ${ }^{3}$ Department of Mathematics and Statistics of the Faculty of Science, Masaryk University, Kotlarska 267/2, 611 37 Brno, Czech Republic \\ ${ }^{4}$ Department of Pathology, Masaryk Memorial Cancer Institute, Zluty kopec 7, 65653 Brno, Czech Republic \\ ${ }^{5}$ Department of Radiology, Masaryk Memorial Cancer Institute, Zluty kopec 7, 65653 Brno, Czech Republic
}

Correspondence should be addressed to Oldřich Coufal; oldrich.coufal@gmail.com

Received 13 February 2014; Revised 21 June 2014; Accepted 25 June 2014; Published 8 July 2014

Academic Editor: Glen Jickling

Copyright (C) 2014 Oldřich Coufal et al. This is an open access article distributed under the Creative Commons Attribution License, which permits unrestricted use, distribution, and reproduction in any medium, provided the original work is properly cited.

\begin{abstract}
Objectives. The aim of the study was to develop a clinical prediction model for assessing the probability of having invasive cancer in the definitive surgical resection specimen in patients with biopsy diagnosis of ductal carcinoma in situ (DCIS) of the breast, to facilitate decision making regarding axillary surgery. Methods. In 349 women with DCIS, predictors of invasion in the definitive resection specimen were identified. A model to predict the probability of invasion was developed and subsequently simplified to divide patients into two risk categories. The model's performance was validated on another patient population. Results. Multivariate logistic regression revealed four independent predictors of invasion: (i) suspicious (micro)invasion in the biopsy specimen; (ii) visibility of the lesion on ultrasonography; (iii) size of the lesion on mammography $>30 \mathrm{~mm}$; (iv) clinical palpability of the lesion. The actual frequency of invasion in the high-risk patient group in the test and validation population was $52.6 \%$ and $48.3 \%$, respectively; in the low-risk group it was $16.8 \%$ and $7.1 \%$, respectively. Conclusion. The model proved to have good performance. In patients with a low probability of invasion, an axillary procedure can be omitted without a substantial risk of additional surgery.
\end{abstract}

\section{Introduction}

In patients with biopsy diagnosis of ductal carcinoma in situ (DCIS) of the breast, an invasive cancer is often found in the definitive surgical resection specimen. The frequency of such upstaging varies in different sets of patients and is usually between $15 \%$ and $40 \%$, with a median value of $26.0 \%$ [1]. Though the incidence of nodal metastases in pure DCIS is rare (3.7\% in a meta-analysis [2]), the role of sentinel lymph node biopsy (SLNB) has been debated, because of the possibility of invasion in the definitive surgical specimen, and there are substantial differences regarding its indication worldwide $[3,4]$. SLNB is not considered a demanding procedure, but there are still some associated costs and inconvenience for the patient. The frequency of lymphoedema occurring three years after SLNB can be as high as $8 \%$ [5]. Thus, routine SLNB in all patients with a biopsy diagnosis of DCIS seems inappropriate. With additional SLNB performed selectively only in cases with subsequent detection of invasion, about one-third of women would be exposed to further surgery. It would be of clinical benefit therefore to stratify patients with a biopsy diagnosis of DCIS before surgery, according to the probability of later upstaging. SLNB could be reserved only for patients with a high probability of having invasive cancer and could otherwise be omitted.

Several authors have attempted to identify variables that could serve as predictors of invasive carcinoma in the resection specimen of patients with DCIS diagnosed by biopsy. Although some have failed to identify any reliable predictor [6], in the majority of the studies variables of predictive value 
have been found; these refer to different clinicopathologic items such as the age of the patient [7], the palpability of the lesion [8-12], the characteristics of the lesion on imaging methods (mass/microcalcifications/others) [8-10, 12-15], the size of the lesion $[7,10,11,14]$, the type of biopsy (core cut/vacuum assisted/excisional) $[7,8,16]$, and histopathologic findings in the biopsy specimen (proportion of affected ducts, grade, suspicious invasion) $[7,8,10,12]$. A more detailed review of the literature on this topic has been published by Brennan et al. [1]. The findings are not fully consistent across the studies and sometimes variables were used that may not be routinely available in the clinical setting (e.g., the size of the lesion on magnetic resonance imaging [11], or that may not be reproducible, owing to local differences in the diagnostic algorithm (e.g., type of biopsy $[7,8,16])$.

The aim of this study was to develop a clinical prediction model for assessing the probability of having invasive cancer in surgical resection specimens in patients diagnosed with DCIS by needle biopsy. The model should be simple to use and include only variables that are generally available in the clinical setting and that are preferably not subject to local differences in the diagnostic procedure.

\section{Methods}

2.1. Test Population and Construction of the Predictive Model. Inclusion criteria for the test population of the study were as follows: (1) DCIS of the breast diagnosed by needle biopsy and (2) resection of the lesion by partial or total mastectomy in the Masaryk Memorial Cancer Institute, Brno, Czech Republic (MOU) between 2006 and 2011. There were no specific exclusion criteria. The participants were identified retrospectively from the database of the MOU.

For the 349 consecutive patients fulfilling the inclusion criteria, clinicopathologic variables available before surgery that, according to published data and the authors' expectations, may be related to the probability of invasive cancer being detected in the definitive surgical specimen were recorded. The variables were collected retrospectively from usual medical records and they are listed with their categories in Table 1.

(i) The age was calculated as a difference between the year of the surgery and the year of the birth date of the patient.

(ii) Assessment of the palpability of the lesion (not palpable/palpable) was at the discretion of the physician performing the physical examination.

(iii) Size of the lesion on mammography was measured by the radiologist as the maximum diameter of the lesion on the standard mediolateral oblique (MLO) or craniocaudal (CC) mammographic views. In multifocal lesions, the diameter of the whole pathologic area was used for calculations, not the size of the largest focus.

(iv) Assessment of the characteristics of the lesion on mammography (presence of microcalcifications and/or a mass or distortion on the mammogram) and visibility of the lesion on ultrasonography were at the discretion of the examining radiologist.

(v) The biopsy device used and the type of image guidance were chosen by the interventional radiologists performing the biopsy. Usually, but not always, lesions visible on ultrasonography were biopsied using the 14-Gauge automated device under ultrasonographic guidance.

(vi) Histologic grade in the biopsy specimen was assessed by the pathologist as the nuclear grade according to the Nottingham grading system for invasive cancer. Lesions with G1 and G2 nuclei were considered nonhigh-grade and lesions with G3 nuclei were considered high-grade. Generally, the high grade DCIS exhibit nuclei with diameters $>2.5$ RBC equivalents, pleomorphism with irregular nuclear contour, coarse clumped and vesicular chromatin, one or more prominent nucleoli, and frequent mitotic figures. Presence or absence of necrosis was not taken into account.

(vii) Suspect (micro)invasion in the biopsy specimen was assessed by the pathologist. It was defined as the presence of suspicious trabecular tumor cell formation in the context of stromal response (e.g., myofibroblastic proliferation and/or dense lymphoid infiltration), which cannot be unequivocally classified as either invasive carcinoma or lobular extension of DCIS, even if the immunohistochemical detection of myoepithelial markers has been performed.

The basic characteristics of patients and their parameters were summarized using frequency tables and descriptive statistics. Association of the considered variables with subsequent detection of invasion in the resection specimen (predictive value) was assessed by univariate analysis, using the Pearson chi-square test and the Fisher exact test for categorical data. Factors showing predictive value in univariate analysis were evaluated by multivariate analysis in cases where all of these factors were available. The cutoff $P$ value used for selecting variables from univariate analysis to multivariate analysis was 0.1 . The sample size was sufficient for regression modeling as we were interested in six potential predictors with more than ten-fold number of outcome events in the analyzed subpopulation. A binary logistic regression with backward elimination method was used to construct a model to assess the probability of having invasive cancer in the definitive resection specimen. The significance level used for a variable to stay in the model was 0.05. To make the model more user-friendly the score number was assigned to each variable as the corresponding Beta coefficient from the multivariate regression model multiplied by 10 . The performance of the model was assessed by concordance statistics and area under ROC curve. The model was subsequently simplified to enable easy sorting of patients into two risk groups, according to the probability of invasion in the definitive specimen. The probability of invasion according to the simplified predictive model was assessed for every 


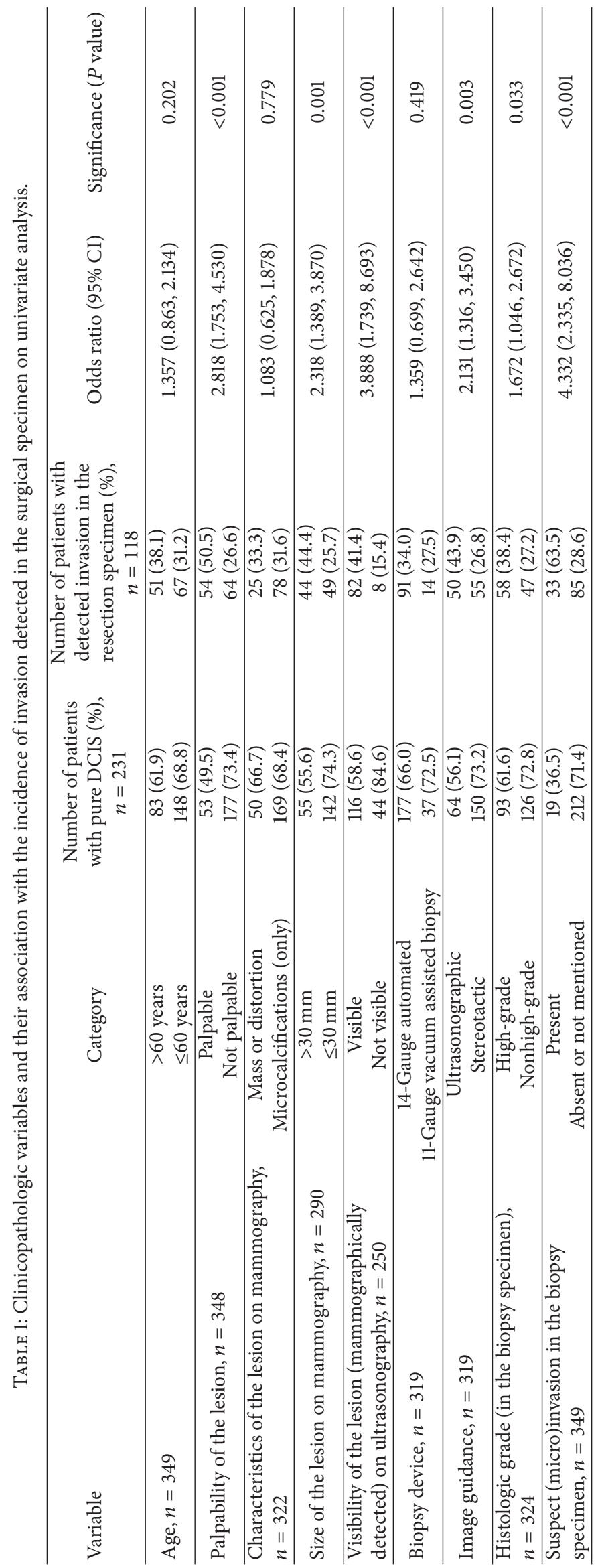


patient in the test population and compared to the real histopathologic finding in the resection specimen.

2.2. Validation of the Model. Subsequently, consecutive patients with DCIS diagnosed by needle biopsy, who underwent resection of the primary lesion at MOU between January 2012 and March 2013 (validation population), were identified. In these patients, the clinicopathologic variables used in the predictive model developed were retrospectively collected from usual medical records. Homogeneity in the test and validation populations was validated using Pearson's chi-square test. The performance of the predictive model was assessed by concordance statistics and area under ROC curve. The probability of invasion according to the simplified model was assessed for every patient of the validation population and compared to the real histopathologic finding in the resection specimen.

Statistical analyses were performed with Statistica (Version 10) and SAS Enterprise Guide (Version 5.1).

\section{Results}

3.1. Test Population and Construction of the Predictive Model. The test population was 349 patients. Their median age was 57 years (range: $22-86$ years) and invasion (or microinvasion) in the resection specimen was detected in 118 cases (33.8\%). The results of univariate analysis assessing the relationship between particular clinicopathologic variables and the detection of invasion are shown in Table 1.

Multivariate logistic regression analysis on 204 patients revealed four independent predictors of invasive cancer on final pathology: (i) suspicious invasion (or microinvasion) in the biopsy specimen; (ii) visibility of the lesion on ultrasonography; (iii) size of the lesion on mammography > $30 \mathrm{~mm}$; (iv) clinical palpability of the lesion.

Using these results, a model to predict the probability of having invasive cancer was developed. The appropriate equation for calculating the probability is

$$
\begin{aligned}
& p=1 \\
& \qquad \begin{aligned}
& \\
& \\
& \quad-0.92 * \text { size }-0.92 * \text { palpable }))^{-1},
\end{aligned}
\end{aligned}
$$

where

(i) $\operatorname{susp} I=1$ if there is present suspect (micro)invasion in the biopsy specimen (otherwise $\operatorname{susp} I=0$ );

(ii) usg $=1$ if the lesion is visible on ultrasonography (otherwise usg $=0$ );

(iii) size $=1$ if the size of the lesion on mammography is $>30 \mathrm{~mm}$ (otherwise size $=0$ );

(iv) palpable $=1$ if the lesion is palpable (otherwise palpable $=0$ ).

According to the user-friendly version of the model, the probability of invasion in an individual case is a function of total score, which is calculated as a sum of specific
TABLE 2: Specific score numbers for variables used in the predictive model; the total score in an individual case is the sum of the corresponding specific score numbers.

\begin{tabular}{lc}
\hline Category of the variable & $\begin{array}{c}\text { Specific score } \\
\text { number }\end{array}$ \\
\hline Suspected (micro)invasion in the biopsy specimen & 13.3 \\
The lesion is visible on ultrasonography & 10.6 \\
The size of the lesion on mammography $>30 \mathrm{~mm}$ & 9.2 \\
The lesion is palpable & 9.2 \\
\hline
\end{tabular}

TABLE 3: The probability of having invasive cancer in the resection specimen (probability of invasion) as a function of total score.

\begin{tabular}{lc}
\hline Total score & Probability of invasion \\
\hline 0 & 0.09 \\
9.8 & 0.20 \\
12.6 & 0.25 \\
15.2 & 0.30 \\
23.7 & 0.50 \\
34.6 & 0.75 \\
\hline
\end{tabular}

TABLE 4: Assessment of the performance of the predictive model in the test population, $n=204$.

\begin{tabular}{lccc}
\hline \multicolumn{3}{c}{ Association of predicted probabilities and observed responses } \\
\hline Percent concordant & 69.2 & Somers' D & 0.511 \\
Percent discordant & 18.0 & Goodman-Kruskal Gamma & 0.586 \\
Percent tied & 12.8 & Kendall's Tau-a & 0.230 \\
Pairs & 9315 & Area under ROC & 0.756 \\
\hline
\end{tabular}

score numbers for corresponding variables. The specific score numbers for each variable used are listed in Table 2 .

The probability of invasion related to the total score is shown in Table 3.

The performance of the predictive model in the test population is shown in Table 4 .

3.2. Simplification of the Model. A probability of invasion equal to 0.25 was set as a cut-off between the "low-risk" and the "high-risk" groups of patients. This cut-off was chosen as clinically relevant with the priority to increase the negative predictive value of the model. The corresponding score number equals 12.6 (see Table 3 ). The presence of suspicious (micro)invasion in the biopsy specimen has a specific score number of 13.3 (see Table 2), which by itself predicts a probability of invasion greater than 0.25 . Therefore, suspicious (micro)invasion is considered a "major risk factor." The specific score numbers for other variables used in the model are less than 12.6 (see Table 2); they were termed "minor risk factors." The presence of only one of these factors gives a probability of invasion under 0.25 . In order to divide the whole population into two risk groups, a simple rule has been set: 
Major risk factor: suspect (micro)invasion in the biopsy specimen

Minor risk factors:

(i) the lesion is visible on ultrasonography

(ii) the size of the lesion on mammography is $>30 \mathrm{~mm}$

(iii) the lesion is palpable

Maximum one minor risk factor present $\rightarrow$ low probability of invasion

Suspected (micro)invasion in the biopsy specimen OR presence of a minimum of two minor

risk factors $\rightarrow$ high probability of invasion

Box 1: A simplified model to predict the probability of invasion in ductal carcinoma in situ of the breast diagnosed by needle biopsy.

TABLE 5: Performance of the simplified model in the test population, $n=204$.

\begin{tabular}{lcccc}
\hline & $\begin{array}{c}\text { Number of patients with } \\
\text { invasion detected in the } \\
\text { resection specimen (\%) }\end{array}$ & $\begin{array}{c}\text { Number of patients with } \\
\text { pure DCIS in the } \\
\text { resection specimen (\%) }\end{array}$ & $\begin{array}{c}\text { Positive predictive } \\
\text { value (95\% CI) }\end{array}$ & $\begin{array}{c}\text { Negative predictive value } \\
(95 \% \text { CI) }\end{array}$ \\
\hline $\begin{array}{l}\text { Low probability predicted } \\
\text { (low-risk group), } n=107\end{array}$ & $18(16.8)$ & $89(83.2)$ & $52.6 \%(42.6,62.5)$ & $83.2 \%(76.1,90.3)$ \\
$\begin{array}{l}\text { High probability predicted } \\
\text { (high-risk group), } n=97\end{array}$ & $51(52.6)$ & $46(47.4)$ & & \\
\hline
\end{tabular}

TABLE 6: Assessment of the performance of the predictive model in the validation population, $n=71$.

\begin{tabular}{lccc}
\hline \multicolumn{3}{c}{ Association of predicted probabilities and observed responses } \\
\hline Percent concordant & 80.6 & Somers' D & 0.705 \\
Percent discordant & 10.1 & Goodman-Kruskal Gamma & 0.777 \\
Percent tied & 9.3 & Kendall's Tau-a & 0.260 \\
Pairs & 918 & Area under ROC & 0.852 \\
\hline
\end{tabular}

(i) if there is a maximum of one minor risk factor present, then the probability of invasion in the resection specimen is low (under 0.25);

(ii) if there is suspicious (micro)invasion in the biopsy specimen, or two or three minor risk factors are present, then the probability of invasion in the resection specimen is high (above 0.25 ).

The rule is highlighted in Box 1 .

The performance of the simplified model in the test population is shown in Table 5.

3.3. Validation of the Simplified Model. The validation population consisted of 71 patients. Their median age was 60 years (range: 32-79 years) and invasion (or microinvasion) in the resection specimen was detected in 17 cases $(23.9 \%)$. Homogeneity in the test and validation population was not rejected at a 0.05 level of significance, suggesting that both populations were comparable in terms of the predictive factors and invasion in the resection specimen. The performance of the predictive model in the validation population is shown in Table 6.

The performance of the simplified model in the validation population is shown in Table 7.

\section{Discussion}

In this study, medical records from 420 patients with DCIS diagnosed by needle biopsy were analysed. Although there were some missing data, still it is one of the largest monoinstitutional studies on this topic to date. It confirmed the predictive value of some clinicopathologic variables in terms of upstaging to invasive cancer. Furthermore, this study has added to the body of research by developing a comprehensive but simple model, with clear-cut results assigning patients into two categories according to their probability of having invasive cancer in the resection specimen.

Contrary to expectations, the predictive value of some variables was not confirmed in the analysed patient population. Most notably, there was no association between invasion in the resection specimen and mammographic appearance of the lesion (mass or distortion and/or microcalcifications). This may have been caused by inappropriate description of the mammographic findings in the usual medical records. However, available mammographic images (from the majority of patients) were reviewed by a single radiologist, with no significant changes in the results. Further, no association was found between upstaging and the thickness of the needle used to obtain the biopsy. The possible association found in other series $[7,8,16]$ may be related to the amount of tissue sampled and histologically examined. In this respect, the number of samples would also be of importance but this variable was not analysed in the present study. The choice of needle may ultimately be influenced by the preferences of the performing radiologist and hence may be biased.

When constructing the model, the variables to be included were carefully considered. Even if the most comprehensive model with six possible variables was built up, the area under the ROC curve for the test population did not go above 0.8 and it was substantially lower in the validation population. Thus, simplicity was preferred, and one variable 
TABLE 7: Performance of the simplified model in the validation population, $n=71$.

\begin{tabular}{lcccc}
\hline & $\begin{array}{c}\text { Number of patients with } \\
\text { invasion detected in the } \\
\text { resection specimen (\%) }\end{array}$ & $\begin{array}{c}\text { Number of patients with } \\
\text { pure DCIS in the } \\
\text { resection specimen (\%) }\end{array}$ & $\begin{array}{c}\text { Positive predictive } \\
\text { value (95\% CI) }\end{array}$ & $\begin{array}{c}\text { Negative predictive value } \\
(95 \% \mathrm{CI})\end{array}$ \\
\hline $\begin{array}{l}\text { Low probability predicted } \\
\text { (low-risk group), } n=42\end{array}$ & $3(7.1)$ & $39(92.9)$ & $48.3 \%(30.1,66.5)$ & $92.9 \%(85.1,100.0)$ \\
$\begin{array}{l}\text { High probability predicted } \\
\text { (high-risk group), } n=29\end{array}$ & $14(48.3)$ & $15(51.7)$ & & \\
\hline
\end{tabular}

was used from each basic diagnostic component: histology of the biopsy specimen (suspected invasion); ultrasonography (visibility); mammography (size); clinical examination (palpability). Different values for the cut-off point used to compare smaller with larger lesions were tested; finally, a value of $30 \mathrm{~mm}$ for the cut-off appeared to be the best with regard to the performance of the model and its clinical use.

Park et al. recently published their nomogram for predicting underestimation of invasiveness in DCIS diagnosed by preoperative needle biopsy [17]. The results are similar to the present ones in some aspects. Their model includes suspicious (micro)invasion in the biopsy specimen, palpability of the lesion, characteristics of the lesion on ultrasonography, and the method of biopsy. The ROC of their model reached 0.75 . However, their nomogram was not suitable for the population used in the present study, as, in most cases, the detailed characteristics of the lesion on ultrasonography (mass and/or calcifications) were not clear from the usual medical records of the study population. Contrary to the findings of Park et al., in the present study population, the method of biopsy showed no predictive value, as discussed above.

There are some limitations to this study. In some patients, the appropriate data were lacking. Not every woman had both basic imaging methods (mammography and ultrasonography) performed before undergoing surgery. Sometimes the ultrasonography was performed after the stereotactic biopsy, so the resulting haematoma made it impossible to decide whether the lesion by itself would have been visible or not. In some patients the exact size of the lesion in millimetres was not recorded in the medical notes. The diameter was measured by reviewing the mammographic images but, in some patients diagnosed outside MOU, these were not available. Sometimes the information about grade was lacking in the histopathologic report of the biopsy and it was not possible to review the biopsy slides from patients with the original histopathologic examination performed outside our institution. Finally, for some cases, even though all the data were completely recorded, it was impossible to decide how some variables should be categorized (e.g., one type of examination performed by two different clinicians with discordant results). But, on the other hand, the real clinical conditions led to construction of a model that is relevant to actual clinical practice and would be applicable to the majority of patients.

\section{Conclusions}

In conclusion, through one of the largest monoinstitutional studies on this topic, a model has been developed to predict the probability of invasive cancer in patients with needle biopsy of DCIS. The model demonstrated good performance in both test and validation population. It is quite simple and gives clear-cut result in terms of low or high probability of invasion. The cut-off value has been selected so that, for patients with a low probability of invasion, an axillary procedure can be omitted without substantial risk of their needing to undergo additional surgery, while patients with a high probability of invasion can be offered up-front SLNB. As the variables used in the model were selected with respect to their clinical use, it should be universally applicable. Validation of the model in independent patient populations is desirable.

\section{Ethical Approval}

The performance of the study complies with the current laws of the Czech Republic. The study protocol was approved by the local ethics committee of the MOU.

\section{Conflict of Interests}

The authors declare that there is no conflict of interests regarding the publication of this paper.

\section{Acknowledgments}

The work was supported by (i) the European Regional Development Fund and the State Budget of the Czech Republic for RECAMO (CZ.1.05/2.1.00/03.0101); (ii) the Ministry of Health of the Czech Republic-DRO (MMCI, 00209805); (iii) the Ministry of Education, Youth and Sports of the Czech Republic, Project A-Math-Net Applied Mathematics Knowledge Transfer Network (CZ.1.07/2.4.00/17.0100). The authors thank Dr. Penny Howes for editing the paper.

\section{References}

[1] M. E. Brennan, R. M. Turner, S. Ciatto et al., "Ductal carcinoma in situ at core-needle biopsy: meta-analysis of underestimation and predictors of invasive breast cancer," Radiology, vol. 260, no. 1, pp. 119-128, 2011.

[2] B. Ansari, S. A. Ogston, C. A. Purdie, D. J. Adamson, D. C. Brown, and A. M. Thompson, "Meta-analysis of sentinel node biopsy in ductal carcinoma in situ of the breast," British Journal of Surgery, vol. 95, no. 5, pp. 547-554, 2008.

[3] J. A. Zujewski, L. C. Harlan, D. M. Morrell, and J. L. Stevens, "Ductal carcinoma in situ: Trends in treatment over time in the 
US," Breast Cancer Research and Treatment, vol. 127, no. 1, pp. 251-257, 2011

[4] G. Farante, S. Zurrida, V. Galimberti et al., "The management of ductal intraepithelial neoplasia (DIN): open controversies and guidelines of the Istituto Europeo di Oncologia (IEO), Milan, Italy," Breast Cancer Research and Treatment, vol. 128, no. 2, pp. 369-378, 2011.

[5] T. Ashikaga, D. N. Krag, S. R. Land et al., "Morbidity results from the NSABP B-32 trial comparing sentinel lymph node dissection versus axillary dissection," Journal of Surgical Oncology, vol. 102, no. 2, pp. 111-118, 2010.

[6] C. H. Lee, D. Carter, L. E. Philpotts et al., "Ductal carcinoma in situ diagnosed with stereotactic core needle biopsy: can invasion be predicted?" Radiology, vol. 217, no. 2, pp. 466-470, 2000.

[7] T. W. F. Yen, K. K. Hunt, M. I. Ross et al., "Predictors of invasive breast cancer in patients with an initial diagnosis of ductal carcinoma in situ: a guide to selective use of sentinel lymph node biopsy in management of ductal carcinoma in situ," Journal of the American College of Surgeons, vol. 200, no. 4, pp. 516-526, 2005.

[8] H. S. Park, S. Park, J. Cho, J. M. Park, S. I. Kim, and B. W. Park, "Risk predictors of underestimation and the need for sentinel node biopsy in patients diagnosed with ductal carcinoma in situ by preoperative needle biopsy," Journal of Surgical Oncology, vol. 107, no. 4, pp. 388-392, 2013.

[9] A. Goyal, A. Douglas-Jones, I. Monypenny, H. Sweetland, G. Stevens, and R. E. Mansel, "Is there a role of sentinel lymph node biopsy in ductal carcinoma in situ? Analysis of 587 cases," Breast Cancer Research and Treatment, vol. 98, no. 3, pp. 311-314, 2006.

[10] S. Schulz, P. Sinn, M. Golatta et al., "Prediction of underestimated invasiveness in patients with ductal carcinoma in situ of the breast on percutaneous biopsy as rationale for recommending concurrent sentinel lymph node biopsy," The Breast, vol. 22, no. 4, pp. 537-542, 2012.

[11] T. Miyake, K. Shimazu, H. Ohashi et al., "Indication for sentinel lymph node biopsy for breast cancer when core biopsy shows ductal carcinoma in situ," American Journal of Surgery, vol. 202, no. 1, pp. 59-65, 2011.

[12] P. Meijnen, H. S. A. Oldenburg, C. E. Loo, O. E. Nieweg, J. L. Peterse, and E. J. T. Rutgers, "Risk of invasion and axillary lymph node metastasis in ductal carcinoma in situ diagnosed by coreneedle biopsy," British Journal of Surgery, vol. 94, no. 8, pp. 952956, 2007.

[13] T. A. King, G. H. Farr Jr., G. J. Cederbom et al., "A mass on breast imaging predicts coexisting invasive carcinoma in patients with a core biopsy diagnosis of ductal carcinoma in situ," The American Surgeon, vol. 67, no. 9, pp. 907-912, 2001.

[14] M. F. Dillon, E. W. McDermott, C. M. Quinn, A. O’Doherty, N. O'Higgins, and A. D. K. Hill, "Predictors of invasive disease in breast cancer when core biopsy demonstrates DCIS only," Journal of Surgical Oncology, vol. 93, no. 7, pp. 559-563, 2006.

[15] J. Leikola, P. Heikkilä, M. Pamilo, K. Salmenkivi, K. Von Smitten, and M. Leidenius, "Predicting invasion in patients with DCIS in the preoperative percutaneous biopsy," Acta Oncologica, vol. 46, no. 6, pp. 798-802, 2007.

[16] E. A. Mittendorf, C. A. Arciero, V. Gutchell, J. Hooke, and C. D. Shriver, "Core biopsy diagnosis of ductal carcinoma in situ: an indication for sentinel lymph node biopsy," Current Surgery, vol. 62 , no. 2, pp. 253-257, 2005.
[17] H. S. Park, H. Y. Kim, S. Park, E.-K. Kim, S. I. Kim, and B.W. Park, "A nomogram for predicting underestimation of invasiveness in ductal carcinoma in situ diagnosed by preoperative needle biopsy," The Breast, vol. 22, no. 5, pp. 869-873, 2013. 


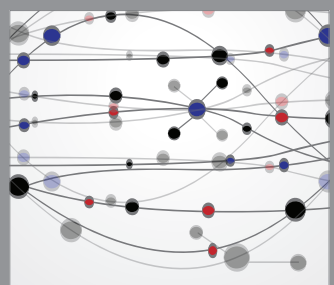

The Scientific World Journal
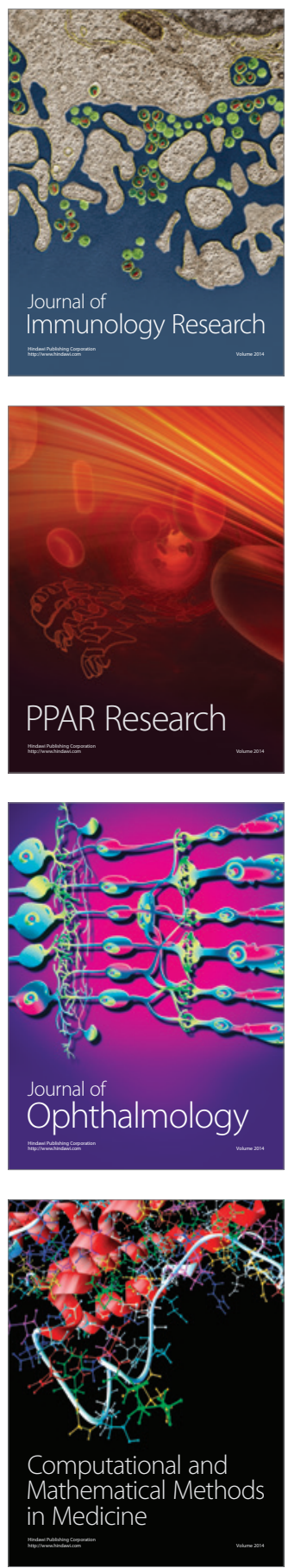

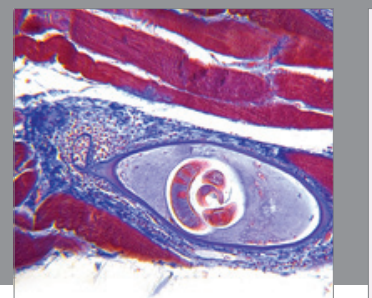

Gastroenterology

Research and Practice
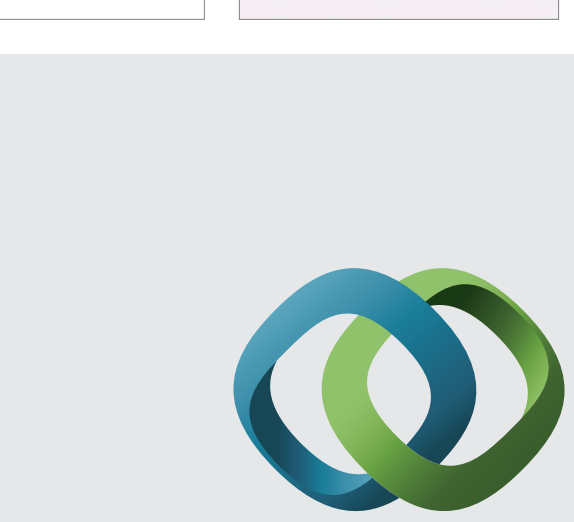

\section{Hindawi}

Submit your manuscripts at

http://www.hindawi.com
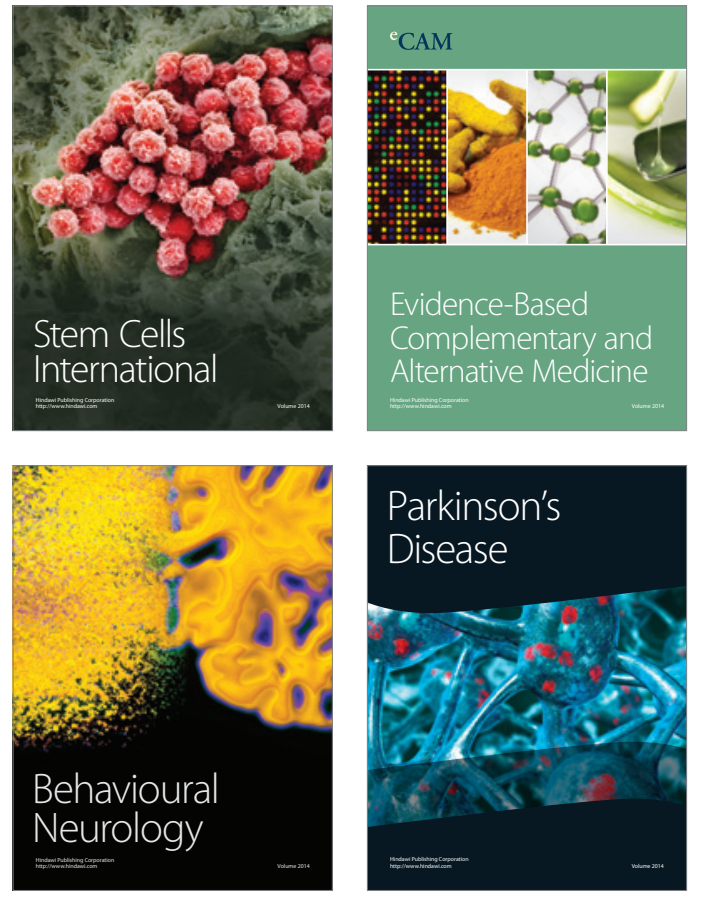
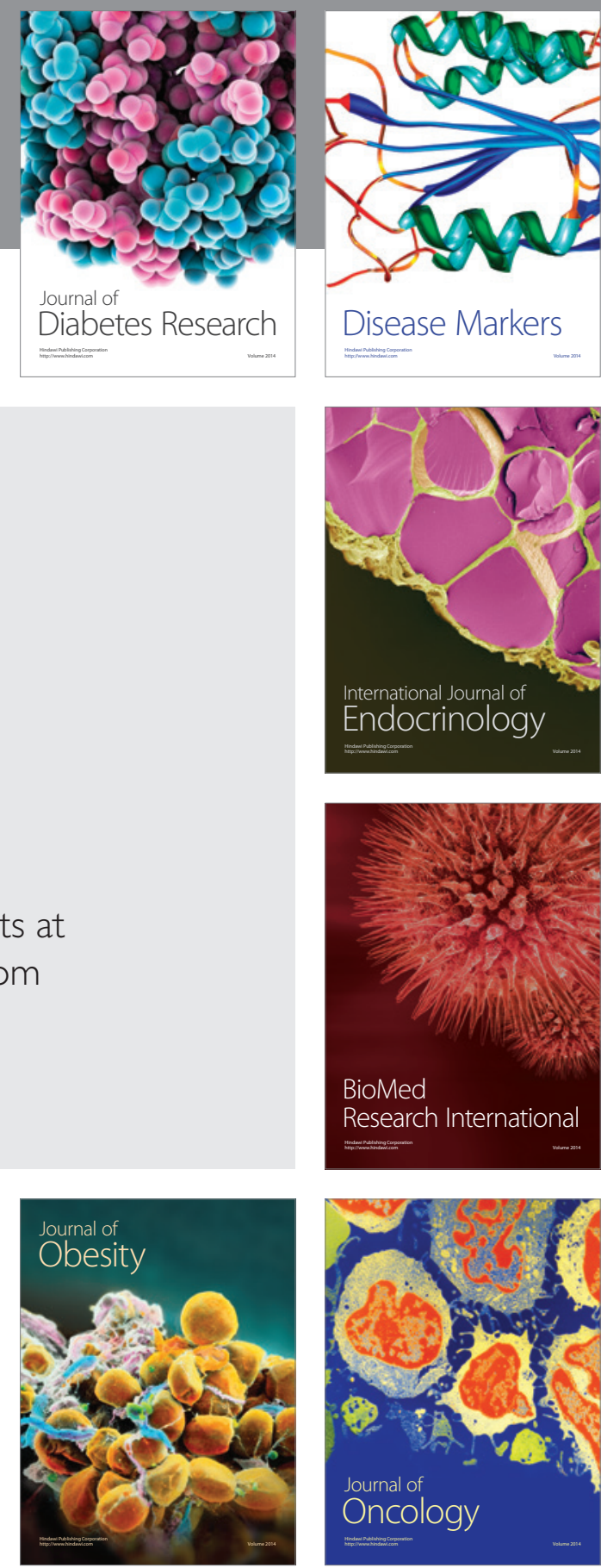

Disease Markers
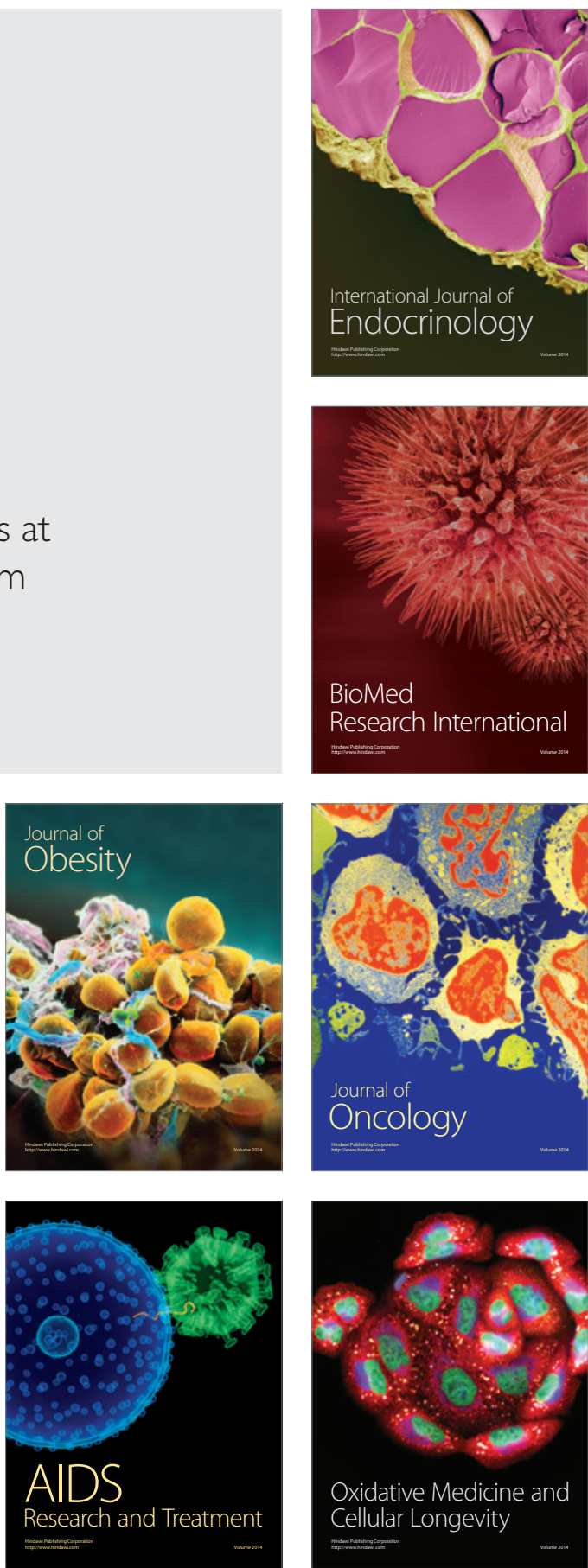